\title{
Clearing the Air from Geneva
}

from a Correspondent

THE second World Administrative Radio Conference for Space Telecommunications, organized by the International Telecommunication Union, was held in Geneva from June 7 to July 17,1971 , and was attended by some 650 delegates from 100 nations. The decisions taken at the first such conference in 1963 have stood the test of time, but developments in space technology and radio astronomy have made new international agreements necessary to cater for the needs of the next decade.

The demand for radio frequencies shows no signs of abating, and as there is a limit to the extent of the radio spectrum which can be exploited the same frequencies should be used by more than one service whenever this is technically feasible. Much of the discussion, and most of the difficulties in reaching agreements, were concerned with problem sharing. As technology advances some of the increased demands can also be met by using frequencies higher than $40 \mathrm{GHz}$, the previous upper limit of the allocated bands, and allocations have now been made up to $275 \mathrm{GHz}$.

Thère has been a remarkable expansion in satellite communications since 1963, and the next decade is expected to see the implementation of satellite services for aeronautical and maritime navigation and communication, for direct broadcasting to the public (at least on a communal basis), for the study of the Earth's resources, and for monitoring the environment. It is also becoming clear that recent discoveries by radio astronomers are providing new insight into the fundamental physics of matter, and that greater protection from interference in several frequency bands is necessary for this important branch of science to develop.

Most of the work of the conference was organized in three committees. The first was concerned with the administrative procedures by which international negotiations may take place as services are introduced or expanded. It has been necessary to revise the procedures laid down in 1963, particularly as the greater emphasis on frequency-sharing calls for clear and more comprehensive procedures by which different countries may coordinate their activities. An interesting departure from custom is that there is now provision for the adoption of new technical criteria on frequency sharing when these are recommended by the International Radio Consultative Committee (CCIR, an advisory committee of the ITU) without waiting for another administrative conference. Countries will have the option of retaining the existing criteria or adopting the revised criteria when these become available.

The second committee was concerned with establishing the technical criteria such as limits of radiated power and power flux densities which are considered necessary to enable services to share frequencies without mutual interference. This committee also provided the technical data needed first for calculating the coordinating distances within which consultation with other countries need take place, and then for calculating the likelihood of interference, and the necessary separation distances, taking into account the actual radio links in operation or planned. Agreement on comprehensive technical standards, in the available time, was made possible only by the thorough preparatory work carried out in February 1971 by a special joint meeting of relevant Study Groups of the CCIR in Geneva, and before that by a CCIR working party in Nice in December 1970, which assembled the latest data on radio propagation necessary to enable the more general studies to proceed.

Since 1963 it has become clear that the future development of space communications is vitally dependent on the exploitation of geostationary satellites, which remain fixed in relation to the surface of the Earth. The technical committee therefore gave considerable attention to the need for the efficient use of the geostationary satellite orbit, and the criteria which must be adopted to avoid interference between links using different satellites in this orbit, and between these links and terrestrial services.

Although the preparation of administrative procedures and technical criteria is fundamental to the development of communications, it is the frequency allocations themselves which will excite the widest interest. The revision of the frequency allocation tables was the task of the third committee, and some difficult problems were encountered. Although space services can be operated over a wide range of frequencies, certain parts of the spectrum have marked advantages and are in heavy demand. One such range, for example, is in the region of $2-3 \mathrm{GHz}$; wide bandwidths are not available at much lower frequencies, and propagation at much higher frequencies can be seriously affected by adverse meteorological conditions. This desirable part of the spectrum, which is already used extensively for terrestrial services, is also in demand for Earth exploration satellites, for broadcasting satellites, and for increased bandwidths in space research. An acceptable compromise was finally achieved, by which it should be possible for these services to share certain bands, provided that there are only a few Earth stations for space research and Earth exploration, that the power flux density from broadcasting satellites is kept below an agreed limit, and that full consultation takes place before any new system is established. Similar problems in other parts of the spectrum were treated in similar ways.

The final agreements on frequency allocations are inevitably the result of compromise, and operators of most services will be disappointed that not all their hopes were realized. It has not been possible to make new primary allocations for space research, except in the newly-allocated part of the spectrum above $40 \mathrm{GHz}$, but several special provisions have been made which will, it is hoped, enable the necessary arrangements for specific operations to be made by negotiation. The basis of this philosophy is that successful operation of a few Earth stations in the space research service, even though a high degree of protection is required, need not inhibit the use of the same frequencies for other services in other parts of the world.

Radio astronomy has received improved status in several bands, and has obtained a new exclusive high frequency band near $22 \mathrm{MHz}$ and new bands above $40 \mathrm{GHz}$. There is increased recognition of the importance of bands needed for observing natural line emissions. On the other hand, the expected increase in transmissions from satellites, for all purposes, constitutes a threat to radio astronomy which cannot be avoided by locating observatories in remote areas. As an example, there is now an allocation for broadcasting satellites in a band adjacent to the exclusive radio astronomy band at 2,690-2,700 $\mathrm{MHz}$. Careful planning and much goodwill is needed if radio astronomers are to be able to use this band without danger of serious interference.

The new regulations will come into force, formally, on January 1, 1973. But as they reflect the established policies of most countries, many of the provisions will already have been adopted for practical purposes, and others will no doubt be followed well in advance of the formal date of implementation. 